\title{
Analysis of Arginine and Lysine Methylation Utilizing Peptide Separations at Neutral pH and Electron Transfer Dissociation Mass Spectrometry
}

\author{
Ambrosius P. L. Snijders, ${ }^{a}$ Ming-Lung Hung, ${ }^{\text {b }}$ Stuart A. Wilson, ${ }^{\text {b }}$ and \\ Mark J. Dickman ${ }^{a}$ \\ ${ }^{a}$ Biological and Environmental Systems, Department of Chemical and Process Engineering, University of \\ Sheffield, Sheffield, United Kingdom \\ ${ }^{\mathrm{b}}$ Department of Molecular Biology and Biotechnology, University of Sheffield, Sheffield, United Kingdom
}

\begin{abstract}
Arginine and lysine methylation are widespread protein post-translational modifications. Peptides containing these modifications are difficult to retain using traditional reversed-phase liquid chromatography because they are intrinsically basic/hydrophilic and often fragment poorly during collision induced fragmentation (CID). Therefore, they are difficult to analyze using standard proteomic workflows. To overcome these caveats, we performed peptide separations at neutral $\mathrm{pH}$, resulting in increased retention of the hydrophilic/basic methylated peptides before identification using MS/MS. Alternatively trifluoroacetic acid (TFA) was used for increased trapping of methylated peptides. Electron-transfer dissociation (ETD) mass spectrometry was then used to identify and characterize methylated residues. In contrast to previous reports utilizing ETD for arginine methylation, we observed significant amount of side-chain fragmentation. Using heavy methyl stable isotope labeling with amino acids in cell culture it was shown that, similar to CID, a loss of monomethylamine or dimethylamine from the arginine methylated side-chain during ETD can be used as a diagnostic to determine the type of arginine methylation. CID of lysine methylated peptides does not lead to significant neutral losses, but ETD is still beneficial because of the high charge states of such peptides. The developed LC MS/MS methods were successfully applied to tryptic digests of a number of methylated proteins, including splicing factor proline-glutamine-rich protein (SFPQ), RNA and export factor-binding protein 2 (REF2-I) and Sul7D, demonstrating significant advantages over traditional LC MS/MS approaches. (J Am Soc Mass Spectrom 2010, 21, 88-96) (c) 2010 American Society for Mass Spectrometry
\end{abstract}

\begin{abstract}
A rginine and lysine methylation are common protein post-translational modifications (PTMs) involved in transcriptional regulation, DNA repair, RNA processing, and signal transduction [1-4]. With the recent identification of enzymes capable of demethylating lysine and arginine residues [5-7], and the observation that arginine methylation on histones can be antagonized enzymatically (via deimination of arginine to citrulline) $[8$, 9], it seems likely that methylation can contribute to the dynamic control of biological processes [10]. Protein arginine N-methyltransferases (PRMTs) catalyze the post-translational transfer of a methyl group from the donor S-adensoyl-L-methioinine (SAM) to arginine residues $[4,11]$. Three forms of arginine methylation have been described $\mathrm{N}^{\mathrm{G}}$-monomethylarginine, $\mathrm{N}^{\mathrm{G}} \mathrm{N}^{\mathrm{G}}$-dimethylarginine (asymmetric dimethylarginine aDMA), and $\mathrm{N}^{\mathrm{G}} \mathrm{N}^{\prime} \mathrm{G}_{-}$ dimethylarginine (symmetric dimethylarginine sDMA). Ly-
\end{abstract}

Address reprint requests to Dr. M. J. Dickman, Department of Chemical and Process Engineering, University of Sheffield, Sheffield S3 7RD, UK. E-mail: m.dickman@sheffield.ac.uk sine can be progressively methylated by lysine or histone methyltransferases to give $\varepsilon$-N-monomethyllysine, $\varepsilon$-N-dimethyllysine, or $\varepsilon$-N-trimethyllysine. In addition to lysine methylation in eukaryotes, it is increasingly clear that lysine methylation is abundant throughout the prokaryotic kingdom [12-14].

Traditionally, protein methylation is detected by Edman sequencing, radioactively by using the tritiated methyltransferase cofactor SAM or via immuno-detection using methylation specific antibodies. Arginine dimethyl antibodies have also been used to enrich for arginine dimethylated proteins [15]. Unfortunately many of these approaches are either aspecific or fail to identify the site and the type of methylation. Mass spectrometry has become the main analytical tool in protein identification and also in the discovery and characterization of PTMs. In some cases, the characteristic fragmentation properties of peptides containing PTMs, such as neutral losses or specific reporter masses, can be exploited. Precursor ion scanning is used to study arginine methylation since two side-chain fragments of DMA, the 
dimethylammonium ion (46.06 Da) and dimethylcarbodiimidium ions $(71.06 \mathrm{Da})$ can be used as specific reporters for arginine methylation [16-18]. Dimethylcarbodiimidium is produced from both aDMA and sDMA, but generally more strongly for sDMA.

In addition, side-chain fragmentation of arginine methylated peptides can often be observed as neutral losses in MS/MS spectra and therefore can be used to determine the type of methylation. A neutral loss of monomethylamine (31.04 Da) is specific for MMA and sDMA, dimethylamine (45.05 Da) for aDMA, and dimethylcarbodiimidium (70.05 Da) for aDMA and sDMA $[18,19]$. Precursor ion scanning is also used to detect the immonium ions of lysine mono- and dimethylation (98.1, $112.1 \mathrm{Da})$. These approaches have been extensively applied for the characterization of histone PTMs $[20,21]$. Despite the development of these approaches, standard LC-MS workflows for arginine and lysine methylated peptides still suffer from a number of disadvantages. Arginine and lysine methylation lead to missed trypsin cleavage sites and arginine methylation predominantly occurs in conserved glycine/arginine rich sequences called GAR or RGG motifs [22, 23]. Methylated peptides therefore frequently contain internal hydrophilic residues, which makes them difficult to capture on hydrophobic stationary phases employed in reversed-phase LC-MS.

Moreover, peptides with internal basic residues often generate poor collision induced (CID) spectra. This is most likely because basic residues sequester protons, thereby reducing their mobility along the peptide backbone and preventing dissociation. As described above, side-chain fragmentation can be exploited for diagnostic purposes. However, reduced fragmentation along the peptide backbone will ultimately compromise the amount of sequence information that can be obtained from such spectra.

Electron-transfer dissociation (ETD) is an alternative fragmentation technique related to electron capture dissociation (ECD) that promotes cleavage of $\mathrm{N}-\mathrm{C}_{\alpha}$ bonds in the peptide backbone resulting in fragment ions of the type $c^{\prime}$ and $z^{\prime} \cdot$ [24]. In general, ETD is particularly effective for highly charged peptides and is less susceptible to side-chain fragmentation compared with CID. Therefore, ETD has become a popular method to study labile post-translational modifications, such as phosphorylation, glycosylation, nitrosylation, sulfonation etc. [25]. ETD and ECD have been extensively used in the study of histone modifications [2629]. Using these techniques, it is possible to obtain extensive sequence information on large peptides and even intact proteins in an approach referred to as top-down sequencing [26-29]. Recently, ETD was also employed for the improved analysis of arginine methylation of synthetic peptides [30] and the identification of symmetric dimethylation of PIWI proteins [31].

Here we use a variety of methylated proteins to generate complex mixtures of methylated peptides that are used to optimize the chromatographic conditions and to study their fragmentation behavior during LCMS. Myelin basic protein (MBP) and the GAR domain containing human proteins splicing factor, proline- and glutamine-rich protein (SFPQ), RNA and export factorbinding protein 2 (REF2-I), and transcriptional coactivator Aly/REF (THOC4) were used to generate peptides containing MMA, aDMA, and sDMA.

The highly basic archaeal chromatin associated proteins Cren7 and Sul7D from the archaeon Sulfolobus solfataricus were digested to generate mixtures of lysine mono- and dimethylated peptides.

\section{Experimental}

\section{Chemicals}

Acetonitrile (LC MS grade), water (HPLC grade), formic acid (FA, HPLC grade), trifluoroacetic acid (TFA, HPLC grade), ammonium formate (MS grade) were obtained from Fisher Scientific, Loughborough, UK. All other chemicals were obtained from Sigma, Poole, UK.

\section{Cell Growth and Stable Isotope Labeling}

For the stable isotope labeling with amino acids in cell culture (SILAC) experiments, HEK 293T cells were grown on DMEM medium supplemented with $\mathrm{CD}_{3}$ methionine and $10 \%$ FCS (10 kDa MW cut-off) in a $37^{\circ} \mathrm{C}$ incubator and $5 \% \mathrm{CO}_{2}$. FCS and DMEM without lysine, arginine, and methionine were obtained from Biosera (Ringmer, UK). $\mathrm{CD}_{3}$ labeled methionine was obtained from Cambridge isotopes (Hook, Hampshire, UK). To initiate the labeling experiments the HEK 293T cells were seeded in a 24 well plate at a concentration of $5 \times 10^{4}$ cells $/ \mathrm{mL}$. Cells corresponding to one well were expanded into a $10 \mathrm{~cm}$ dish and harvested at $\sim 100 \%$ confluence. The labeling efficiency was analyzed by mass spectrometry and was shown to be complete (data not shown).

\section{Protein Purification and Preparation}

SFPQ was isolated from HeLa nuclear extracts (CILBIOTECH, B700-Mons, Belgium) using Ni-NTA open tube capillary enrichment, by virtue its interacting partner NONO as previously described [32]. A FLAGtagged REF2-I construct was transiently transfected into 293T cells, purified under stringent conditions using FLAG-agarose as previously described [33]. In each case the protein purifications were analyzed using SDS PAGE confirming proteins were purified to greater than 90\% (see Supplementary Figure 1, which can be found in the electronic version of this article). REF2-I was purified to $0.1 \mathrm{mg} / \mathrm{mL}$. No other significant proteins could be detected using SDS-PAGE, Coomassie staining. SFPQ co-purified with its binding partner NONO in a ratio $1: 1$ on the basis of Coomassie staining. The total protein concentration was estimated at $0.5 \mathrm{mg} /$ mL. THOC4 was identified from a HeLa nuclear extract 
tryptic digest. Sul7D and Cren7 were purified from $S$. solfataricus following cell growth, acid extraction, and RP HPLC separation. Bovine myelin basic protein was obtained from Sigma UK. All proteins (500 ng) were digested with trypsin (Sigma proteomics grade, 0.1-200 $\mathrm{ng}$ ) in $100 \mathrm{mM}$ ammonium bicarbonate, $20 \%$ acetonitrile at $37^{\circ} \mathrm{C}$ for $1-6 \mathrm{~h}$. The reactions were quenched by the addition of $0.1 \%$ TFA. The samples were subsequently dried under vacuum and resuspended in $0.1 \%$ final concentration of TFA. Six $\mu \mathrm{L}$ was used for LC-MS/MS analysis.

\section{ESI Trap MS/MS Analysis}

Peptides were separated using an Ultimate 3000 capillary liquid chromatography system (Dionex, Camberley, UK), using a $75 \mu \mathrm{m}$ i.d. $\times 15 \mathrm{~cm}$ PepMap reverse phase column (Dionex, UK). Linear gradient elution was performed using buffer A ( $0.1 \%$ formic acid) and buffer B $(0.1 \%$ formic acid, $95 \%$ acetonitrile) starting from $5 \%$ buffer B to $40 \%$ over $40 \mathrm{~min}$ at a flow rate of $300 \mathrm{~nL} / \mathrm{min}$. Direct injection analysis was performed using Atlantis C18 capillary column $300 \mu$ m i.d. $\times 15$ $\mathrm{cm}$ (Waters, Elstree, UK). Linear gradient elution was performed starting at buffer $5 \%$ buffer B to $40 \%$ buffer B over $40 \mathrm{~min}$ at a flow rate of $2 \mu \mathrm{L} / \mathrm{min}$. Separations at neutral $\mathrm{pH}$ were performed using a linear gradient elution; buffer A (20 mM ammonium formate $\mathrm{pH} 7.0)$, buffer B $(20 \mathrm{mM}$ ammonium formate $\mathrm{pH} 7.0,95 \%$ acetonitrile), starting at $5 \%$ buffer B to $95 \%$ buffer B over $40 \mathrm{~min}$ at a flow rate of $2 \mu \mathrm{L} / \mathrm{min}$.

MS/MS analysis was performed using a HCT Ultra PTM Discovery instrument (with Esquire control, data analysis, and biotools for automated data acquisition and processing (Bruker Daltonics, Bremen, GmbH, Germany). MS1 profile scans $(m / z$ 300-1800) were acquired in standard enhanced positive mode and were followed by two CID and ETD fragmentation experiments in alternating fashion in ultrascan mode $(\mathrm{m} / \mathrm{z} 100-1800)$. For fragmentation, the trap was loaded to a target value of 200,000 with a maximum accumulation time of 200 $\mathrm{ms}$. The precursor isolation width was set to 4.0 and singly charged precursors were excluded. For ETD fragmentation, fluoranthene was allowed to accumulate to a target value of 500,000, and the reaction was allowed to proceed for $100 \mathrm{~ms}$. ETD analysis was performed both with and without smart decomposition (CRCID) of doubly charged ions.

Profile data were then processed into peak list by data analysis using the following settings. The apex peak finder algorithm was used for peak detection using a peak width at half maximum (FWHM) of 0.1 $\mathrm{m} / \mathrm{z}$, a signal to noise ration $(\mathrm{S} / \mathrm{N})$ of 1.0 , a relative to base peak intensity of $0.1 \%$, and an absolute intensity threshold of 10. Spectra were deconvoluted with charge state deconvolution from fragment spectra allowed. Peak lists were then exported as Mascot Generic Files (MGF) and searched using Mascot 2.2 server (Matrix Science, London, UK) with ETD fragmentation rules specified. Mass accuracies were set to $1.8 \mathrm{Da}$ in MS1 mode and 0.6 Da in MS2 mode. Methionine oxidation, dimethylation and methylation of arginine or lysine were used as variable modifications in searches against the Swissprot database (Swiss-Prot Release 51.6, February 6, 2007, 257964 sequences). For heavy methyl SILAC, the masses of the modifications were increased accordingly. Peptides scoring $<25$ were automatically rejected whereas all modified peptides scoring $>25$ were subjected to manual verification with the aid of Biotools within Compass software.

\section{Results and Discussion}

\section{Development of Reversed-Phase Separations of Arginine Methylated Peptides}

Preliminary LC-MS analysis of SFPQ and REF2-I tryptic digests failed to identify arginine methylated peptides, which suggested that peptides were not retained on the C18 stationary phase. In an approach to retain the basic peptides on the $\mathrm{C} 18$ trapping cartridge, the strong ion-pairing reagent trifluoroacetic acid (TFA) was used as an alternative to formic acid (FA) for peptide trapping. After trapping, the peptides were eluted onto the analytical column and separated by a gradient of mobile phases containing the mass spectrometry compatible ion-pairing reagent FA. Under these conditions the hydrophobicity of the ion-paired arginine methylated peptides was increased sufficiently to allow for their retention on the trap column and subsequent detection by mass spectrometry.

These successful experiments indicated that standard proteomic workflows are not optimal for methylated peptides and lead us to investigate alternative methods to increase their retention by changing the $\mathrm{pH}$ of the buffer system. Peptide chromatography under nonacidic conditions has previously been reported and could be advantageous because it alters their selectivity and retention behavior [34, 35]. However, under alkaline conditions, predominantly singly charged peptides with poor fragmentation properties during tandem MS spectra are generated [34]. Furthermore, silica based stationary phases are not stable at high $\mathrm{pH}$ leading to a reduced column lifetime. Therefore, peptide separations at neutral $\mathrm{pH}$ using ammonium formate buffered solvents were explored. In addition, a C18 stationary phase with enhanced retention of polar analytes compared to conventional C18 stationary phases was used (see Experimental). The extracted ion chromatograms from selected identified methylated peptides from REF2-I under either acidic ( $\mathrm{pH} 2.3,0.1 \%$ formic acid) or neutral $\mathrm{pH}(\mathrm{pH} 7.0,20 \mathrm{mM}$ ammonium formate) are shown in Figure 1a and b, respectively.

Under acidic conditions, peptides were retained to the trap column because they were adjusted to $0.1 \%$ TFA. All methylated peptides elute in the first half of the gradient (in the first $16 \mathrm{~min}$ ), demonstrating the hydrophilic nature of such peptides. Moreover, the pep- 

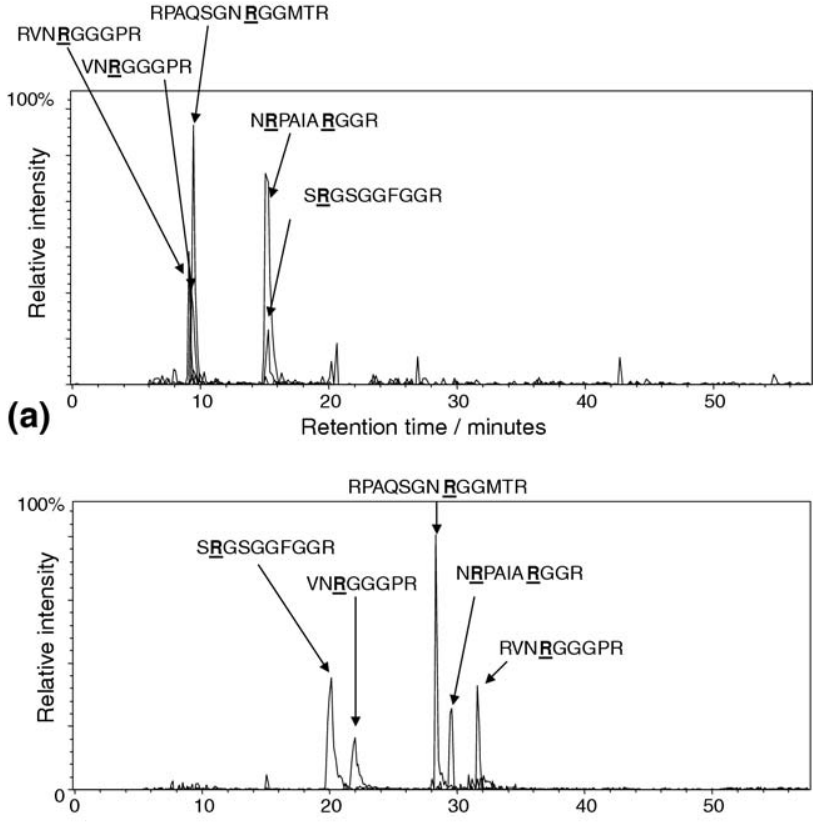

(b)

Retention time / minutes

Figure 1. Extracted ion chromatogram (XIC) of selected methylated peptides generated from REF2-I. (a) Reversed phase chromatography at acidic $\mathrm{pH}(0.1 \%$ formic acid $)$. (b) Reversed phase chromatography at neutral $\mathrm{pH}(20 \mathrm{mM}$ ammonium formate $\mathrm{pH}$ 7.0).

tides (VN(dimeR)GGGPR, RPAQSGN(dimeR)GGMTR, RVN(dimeR)GGGPR) show little or no retention on the analytical column and therefore co-elute, which could have a negative impact on their detection by LC-MS.

At neutral $\mathrm{pH}$ (Figure $1 \mathrm{~b}$ ) and under the same acetonitrile gradient, the methylated peptides elute in the second half of the gradient, therefore demonstrating increased retention on the analytical column. The increased retention of the methylated peptides at neutral $\mathrm{pH}$ facilitates their separation and prevents their coelution. Therefore, neutral $\mathrm{pH}$ separations can enhance the ability of LC-MS to detect methylated peptides.

\section{Arginine Methylation Analysis Using CID and ETD Tandem MS}

Standard mass spectrometry approaches for proteomics rely on the availability of databases containing predicted protein sequences. Compared with the primary amino acid sequence, the nature and localization of PTMs, such as protein methylation, is far more difficult to predict from the genome sequence alone and therefore requires high quality and informative mass spectra. Here, we perform a detailed analysis of the CID and ETD spectra of a variety of arginine and lysine methylated peptides. Figure $2 \mathrm{a}$ and $\mathrm{b}$ show the typical CID and ETD mass spectra of the tetradimethylated peptide SQ(dimeR)GG(dimeR)GGG(dimeR)G(dimeR)GR [M + $3 \mathrm{H}]^{3+}$ from THOC4. As expected, trypsin did not cleave
C-terminal of the dimethylated arginine residues, resulting in a highly charged/basic peptide. CID generated limited backbone cleavage, with a predominant neutral loss of dimethylamine observed (45 Da), thereby indicating the presence of aDMA rather than sDMA. This example illustrates the strength of CID in assigning the type of modification by means of a specific fragment, but also its weakness in failing to generate sufficient backbone fragmentation to allow for sequence determination. In contrast, the ETD spectrum
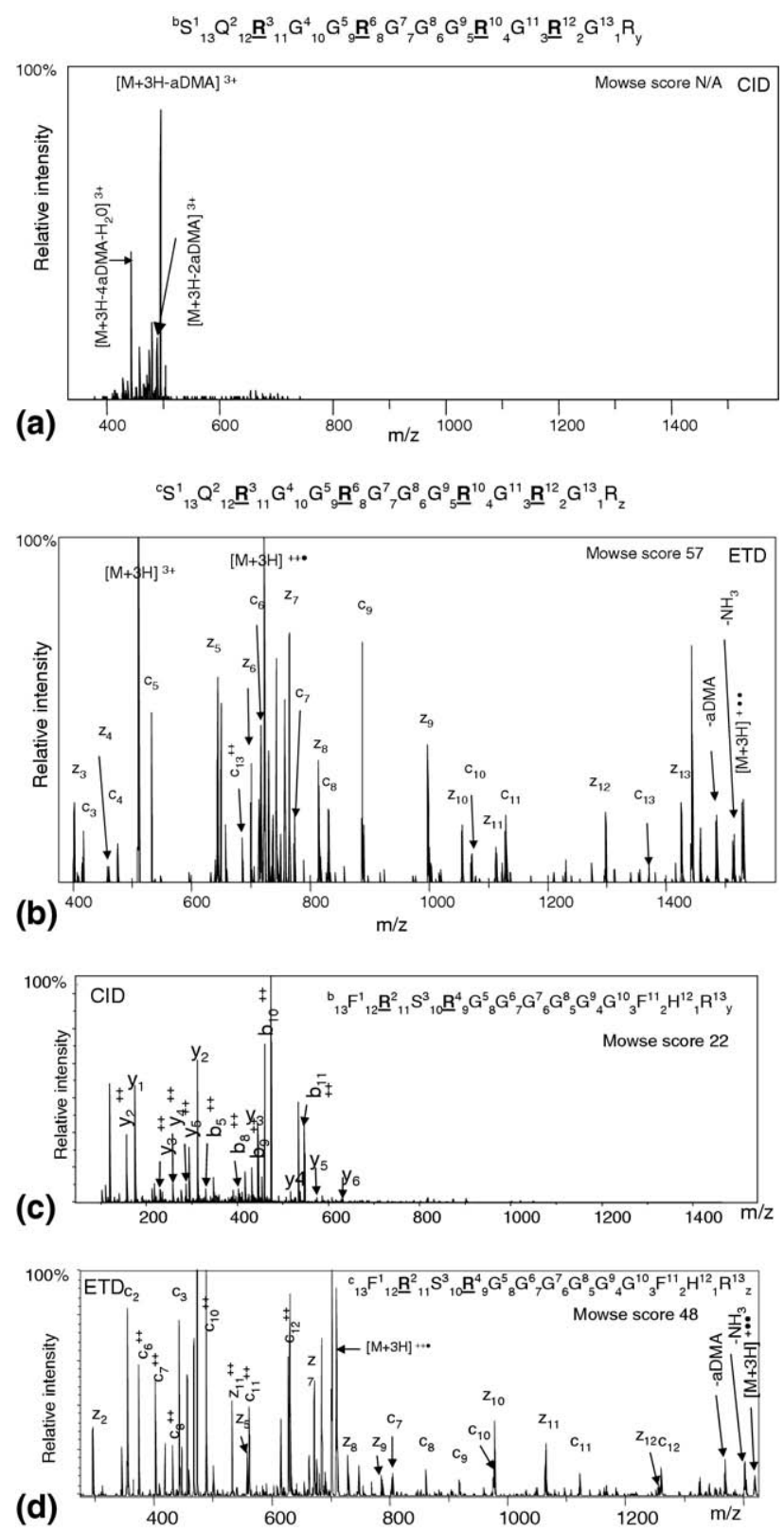

Figure 2. Tandem MS analysis of the methylated peptides. SQ(dimeR)GG(dimeR)GGG(dimeR)G(dimeR)GR $[\mathrm{M}+3 \mathrm{H}]^{3+}$. (a) CID MS/MS fragmentation spectra, (b) ETD MS/MS fragmentation spectra; (c) CID MS/MS analysis of the methylated peptide F(dimeR)S(dimeR)GGGGGGFHR; (d) ETD MS/MS analysis of the methylated peptide F(dimeR)S(dimeR)GGGGGGFHR. The prominent $\mathrm{y}, \mathrm{b}, \mathrm{c}^{\prime}, \mathrm{z}^{\prime} \bullet$ ions are highlighted. 
shows extensive backbone fragmentation with near complete $c^{\prime}$ and $z^{\prime \bullet}$ ion series, enabling confident assignment of the sites of the methylation in this tetradimethylated peptide.

A detailed inspection of the ETD spectrum (Figure $2 \mathrm{~b}$ ) reveals the presence of ions that could be the result of neutral losses typical for arginine methylated peptides, such as a loss of dimethylamine $(-45 \mathrm{Da})$. Further inspection of the ETD spectra of two arginine methylated peptides identified from SFPQ show the consistent improved backbone fragmentation of ETD compared with CID and the presence of neutral losses (see Figure 2c and d, and Supplementary Figure S2 A and B). Despite being considered less prone, ETD has previously been reported to result in a number of uncharacterized and characterized neutral losses such as loss of ammonia and loss of small amounts of phosphoric acid from phosphorylated peptides. In addition, fragmentation of the arginine side chain during ETD has been studied in detail by the group of Turecek to study the basic ETD mechanism. They showed that capture of an electron by the guanidinium group of arginine can result in side-chain rather than backbone dissociation [36]. Recently, Wang et al. observed neutral losses for arginine methylated peptides in CID but did not report such losses from ETD spectra [30].

Here, we investigate whether the neutral losses in ETD can be used directly to determine the type of arginine methylation. Because of the relatively low resolution of the ion trap, we designed experiments to investigate and validate the neutral loss observed in the ETD spectra. Initial MS ${ }^{3}$ experiments were unsuccessful because of low intensities and the presence of isobaric masses in the $[\mathrm{M}]-45 \mathrm{Da}$ region. For this reason we decided to use heavy methyl SILAC to specifically alter the masses of the methylated groups. For heavy methyl SILAC, HEK-293T cells were grown in the presence of heavy methionine $\left(\mathrm{CD}_{3}\right.$-L-methionine). Heavy methio-

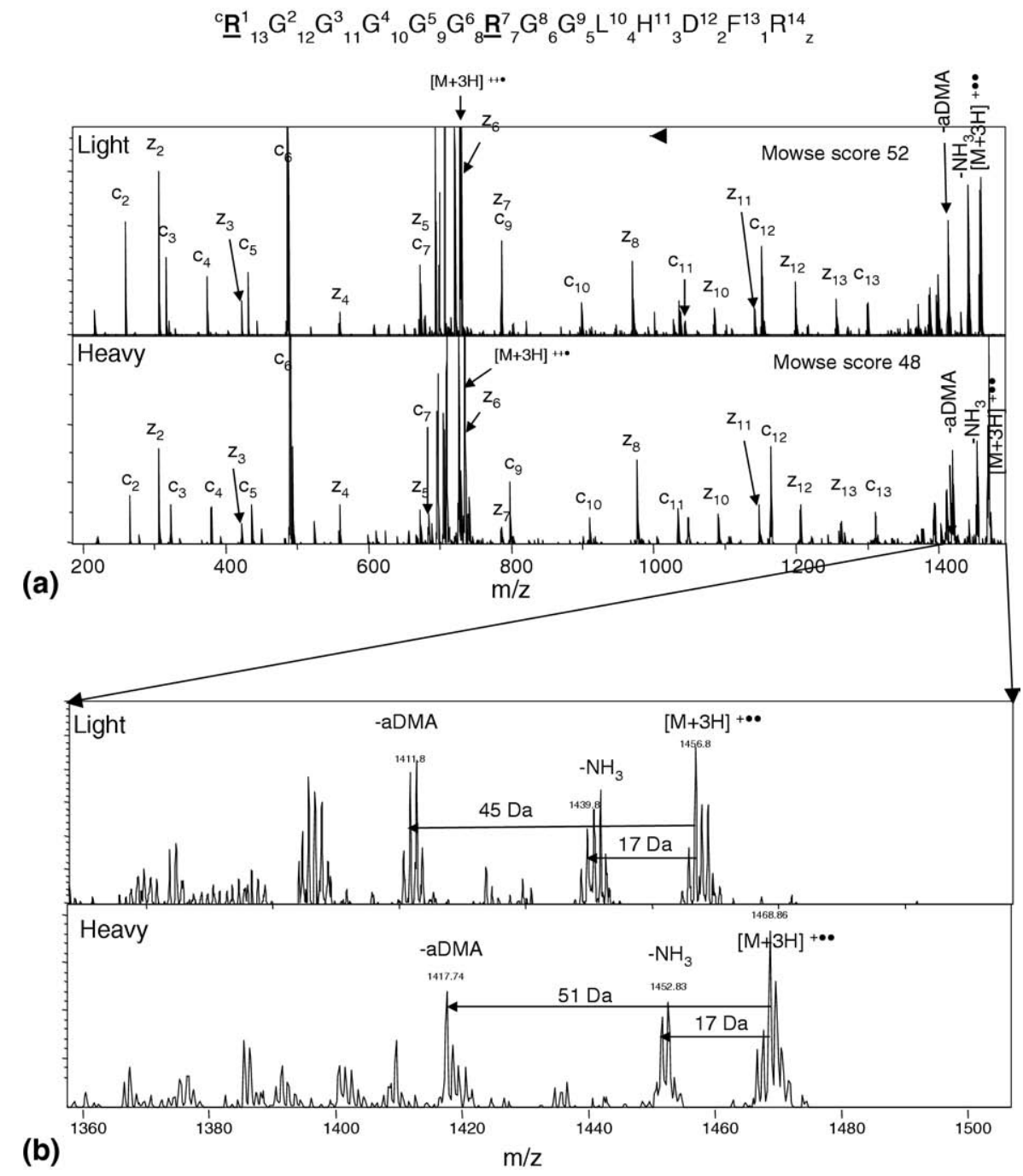

Figure 3. Tandem MS analysis of the heavy methyl labeled peptide (dimeR)GGGGG(dimeR)GGLHDFR. (a) ETD MS/MS analysis of the light and heavy methylated peptides; (b) enhanced view of the neutral losses from the light $[\mathrm{M}+3 \mathrm{H}]^{+} \bullet$ species and heavy $[\mathrm{M}+3 \mathrm{H}]^{+} \bullet$ species. The prominent $\mathrm{c}^{\prime}, \mathrm{z}^{\prime}{ }^{\prime}$ ions and neutral loss of ammonia and dimethylamine are highlighted. 
$N$ (dimeR)PAIA(dimeR)GG(meR)

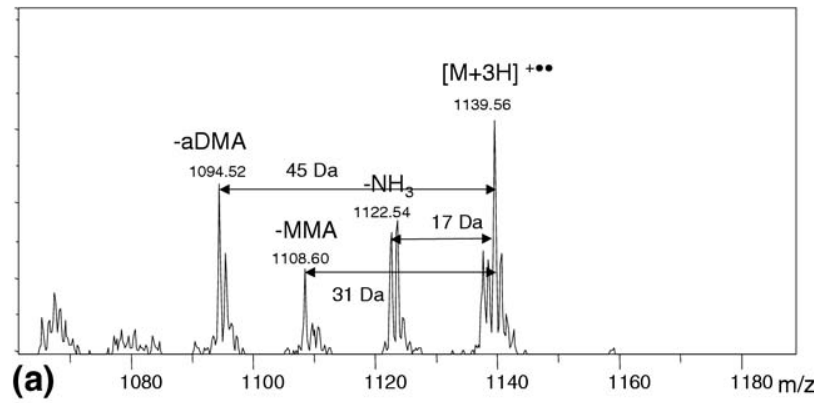

$\mathrm{N}$ (dimeR)PAIA(dimeR)GGR

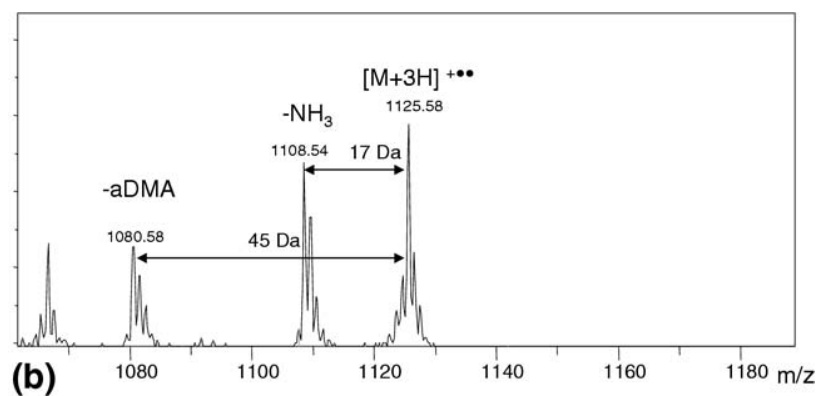

Figure 4. Enhanced view of the neutral loss events from the ETD analysis of methylated peptides; (a) N(dimeR)PAIA(dimeR)GG(meR); (b) N(dimeR)PAIA(dimeR)GGR generated from REF2-I. Neutral losses of ammonia, dimethylamine, and monomethylamine are highlighted.

nine is converted by the cell to heavy SAM, which serves as a universal methyl-group donor in methyl transfer reactions including arginine methylation. The SFPQ/NONO protein complex was purified from these heavy cells. ETD analysis of trypsin digests from SFPQ enabled the identification of the heavy labeled methylated peptides (dimeR)GGGGG(dimeR)GGLHDFR and $\mathrm{F}$ (dimeR)S(dimeR)GGGGGGFHR shown in Figure 3 and Supplementary Figure S3. The tandem MS analysis shows the resulting shift of the corresponding heavy $\mathrm{c}^{\prime}$ and $\mathrm{z}^{\prime}$ ions by an additional $6 \mathrm{Da}$ due to the addition of the heavy dimethyl group $\left(\mathrm{C}_{2} \mathrm{D}_{6}\right)$, providing further evidence for the modifications of these peptides (see Figure $3 \mathrm{a}$ and Supplementary Figure S3A). The observed neutral loss from the $[\mathrm{M}+3 \mathrm{H}]^{+} \bullet$ is highlighted in Figure $3 \mathrm{~b}$ and Supplementary Figure S3B. The spectra show the expected loss of dimethylamine (45 Da) from the light asymmetrically dimethylated peptide. In contrast, the heavy methyl containing peptide generates a neutral loss of $51 \mathrm{Da}$, corresponding to a loss of heavy dimethylamine. The original loss of $45 \mathrm{Da}$ observed in the "light" spectra disappears from the ETD spectra of heavy methyl labeled peptides. These results clearly demonstrate the ability to assign the type of arginine methylation based on specific neutral loss events from the ETD spectra.

Evidence of neutral loss of methylamine (31 Da) from monomethylated peptides was obtained from the peptide NRPAIARGGR from REF2-I. Figure 4 shows the ETD spectra of the two isoforms of this peptide in which R2 and R7 are dimethylated and R9 is monomethylated or unmethylated. The enhanced view shows the neutral loss of both monomethylamine (31.04 Da) and dimethylamine (45.05 Da) from $\mathrm{N}$ (dimeR)PAIA(Rdime)GG(meR) (Figure 4a). In the case of N(dimeR)PAIA(dimeR)GGR the loss of monomethylamine is not observed, whilst the loss of dimethylamine can still be seen (Figure $4 b$ ).

Finally, neutral losses from sDMA were also investigated using the example peptide G(dimeR)GLSLSR obtained from bovine myelin basic protein (Figure 5). Neutral loss of the monomethylamine and dimethylcarbodiimidium were observed in both the CID and ETD spectra, thereby enabling the assignment of symmetric dimethylation of arginine in this peptide, consistent with previous observations [37].

The above discussion has mainly focused on ETD spectra of peptides with charge states $>2+$. It should be noted that a significant portion of methylated peptides
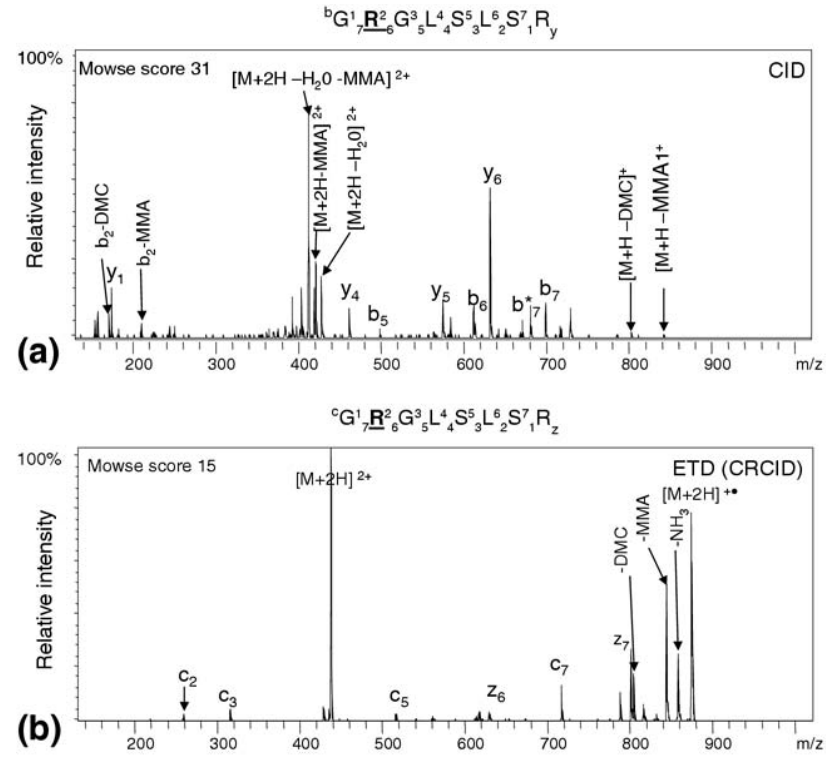

${ }^{\mathrm{c}} \mathrm{T}^{1}{ }_{14} \mathrm{P}^{2}{ }_{13} \mathrm{P}^{3}{ }_{12} \mathrm{~S}^{4}{ }_{11} \mathrm{Q}^{5}{ }_{10} \mathrm{G}^{6}{ }_{9} \mathrm{~K}_{8}^{7} \mathrm{G}_{7}^{8} \mathrm{R}_{6}{ }_{6} \mathrm{G}^{10}{ }_{5} \mathrm{~L}^{11}{ }_{4} \mathrm{~S}^{12}{ }_{3} \mathrm{~L}^{13}{ }_{2} \mathrm{~S}^{14}{ }_{1} \mathrm{R}_{\mathrm{z}}$

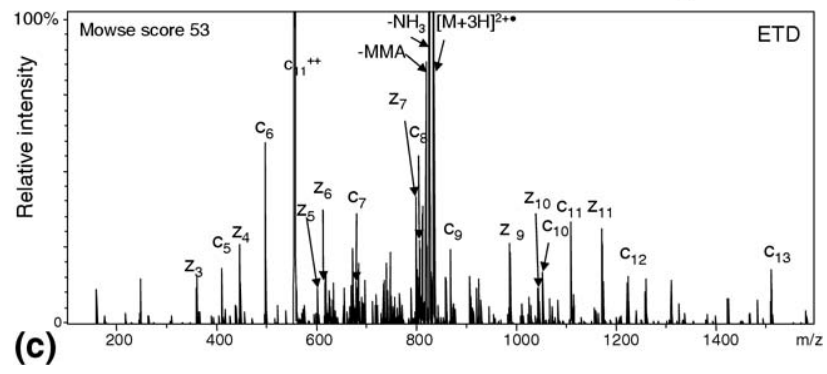

Figure 5. Tandem MS analysis of the methylated peptides generated from myelin basic protein. (a) CID MS/MS analysis of the methylated peptide G(dimeR)GLSLSR; (b) ETD (CRCID) MS/MS analysis of the methylated peptide G(dimeR)GLSLSR. (c) ETD MS/MS analysis of the methylated peptide TPPPSQGKG(dimeR)GLSLSR The prominent $\mathrm{y}, \mathrm{b}, \mathrm{c}^{\prime}, \mathrm{z}^{\prime}{ }^{\prime}$ ions and neutral losses are highlighted. 
such as G(dimeR)GLSLSR shown in Figure 5 can still be observed at a charge state of +2 . As expected in the analysis of such peptides, CID fragmentation generated superior $\mathrm{b}$ and $\mathrm{y}$ ion distribution (Figure $5 \mathrm{a}$ ) compared with the $\mathrm{c}^{\prime}$ and $\mathrm{z}^{\prime}{ }^{\bullet}$ ions generated in the ETD spectra, even with the use of additional ion activation/collision induced dissociation of charge reduced series (CRCID) of the doubly charged peptide using the smart decomposition option (see Figure 5b). Further analysis of myelin basic protein using ETD analysis also enabled the identification and assignment of symmetric dimethylation at the corresponding arginine residue from the larger tryptic peptide TPPPSQGKG(dimeR)GLSLSR (see Figure 5c).

Altogether, these results demonstrate that the highly charged nature of arginine methylated peptides generated from tryptic digests of methylated proteins exhibit favorable fragmentation using ETD in comparison to CID. In addition, CID of arginine methylated peptides mainly results in loss of labile side-chain fragments, whereas ETD is less prone to this type of fragmentation. However, some side-chain fragmentation leading to neutral losses also occurs in ETD, and this is beneficial in determining the type of methylation on the basis of the ETD spectra alone. The neutral losses were observed using both ETD (without smart decomposition) and ETD CRCID (with smart decomposition) and therefore both approaches can be used to determine the type of arginine methylation present.

\section{Lysine Methylation Analysis Using CID and ETD} Tandem MS

Trypsin digestion of Sul7D and Cren7 yielded a number of lysine methylated peptides with internal basic residues. Figure 6a-d show the CID and ETD spectra for the triply charged peptides TPAG(meK)EAELVPEK and TPAG(dimeK)EAELVPEK corresponding to Cren7. In contrast to arginine mono- and dimethylation, no observable neutral loss or side-chain fragmentation was generated during CID of these lysine mono- and dimethylated peptides. Therefore, a sufficient amount of backbone fragmentation could be obtained to allow for peptide identification and to allow for the localization of the methyl groups. Using the CID spectra, it was possible to distinguish between the isobaric candidates TPAG(dimeK)EAELVPEK and TPAG(meK)EAELVPE(meK) (see Figure 6a and b).

The corresponding ETD spectra in Figure $6 \mathrm{c}$ and $\mathrm{d}$ also show comprehensive backbone fragmentation, enabling confident peptide identification and PTM localization. The Mowse scores assigned by Mascot were slightly higher for the ETD spectra compared with the CID spectra, indicating higher information content. In general, we obtained higher Mowse scores using ETD compared with CID for lysine methylated peptides. For Sul7D, the ETD and CID analysis revealed a number of previously identified sites and additional novel sites of monomethyllysine [13, 30, 38] (summarized in Table 1 and Supplementary Figure S4).

(a)

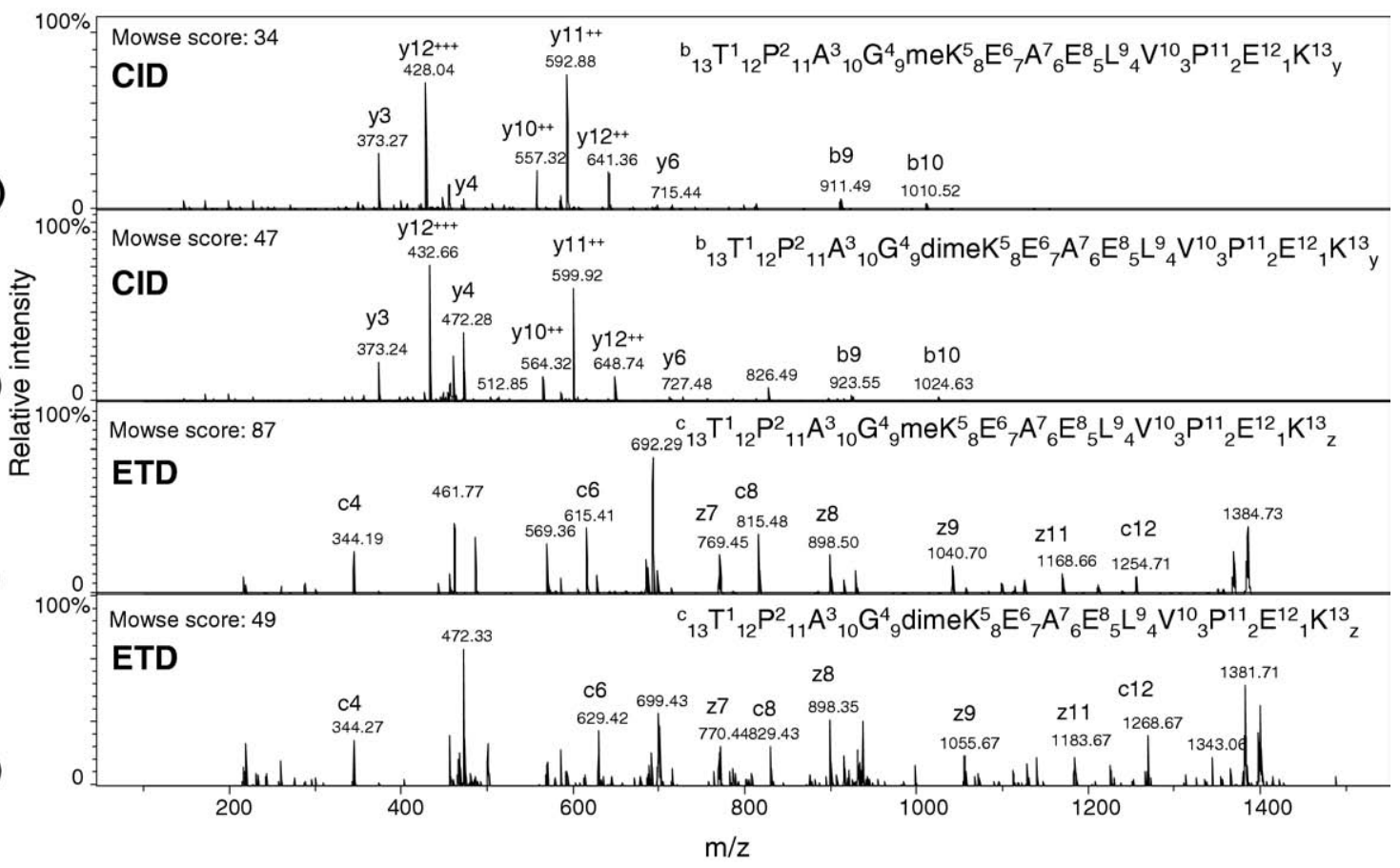

Figure 6. Tandem MS spectra of lysine mono- and dimethylated peptides. (a) CID spectrum of TPAG(meK)EAELVPEK; (b) CID spectrum of TPAG(dimeK)EAELVPEK; (c) ETD spectrum of TPAG(meK)EAELVPEK; (d) ETD spectrum of TPAG(dimeK)EAELVPEK. All spectra were generated from triply charged precursors. 
Table 1. Summary the methylated peptides identified from Sul7D including unambiguous assignment of the site(s) of modification

\begin{tabular}{llcc}
\hline \multicolumn{1}{c}{ Peptide* } & Modification & Charge state & Fragmentation \\
\hline \hline ATVKFK & Methyl (K) & +2 & CID \\
ATVKFKYK & 2 Methyl (K) & +3 & ETD \\
ATVKFKYKGEEKEVDISK & 2 Methyl (K) & +3 & ETD \\
ELLQMLEK & Methyl (K) & +2 & CID/ETD \\
ELLQMLEKQK & 2 Methyl (K) & +3 & ETD \\
ELLQMLEKOKK & 2 Methyl (K) & +3 & ETD \\
DAPKELLQMLEK & Methyl (K) & +3 & CID/ETD \\
FKYKGEEK & Methyl (K) & +3 & ETD \\
FKYKGEEKEVDISK & Methyl (K) & +3 & CID/ETD \\
GAVSEKDAPKELLQMLEK & Methyl (K) & +3 & ETD \\
GAVSEKDAPKELLQMLEK & 2 Methyl (K) & +4 & ETD \\
\hline
\end{tabular}

*Monomethyl groups are highlighted in bold.

The corresponding CID and ETD (CRCID) spectra for the doubly charged TPAG(meK)EAELVPEK peptide only generated a few backbone fragments during ETD (CRCID), whereas the CID spectrum is of considerable better quality (see Supplementary Figure S5). These observations are consistent with previous comparisons of ETD and CID spectra for different charge states. Due to their high basicity, lysine methylated peptides with internal basic residues are predominantly observed at charge states $>+2$. For example, the intensity of the +2 ion for TPAG(dimeK)EAELVPEK was at background level and, therefore, ETD and CID spectra could only be obtained for the +3 ion. Improved LC-MS detection of lysine methylated peptides during ETD is thus mainly due to their relatively high charge state.

\section{Conclusions}

The analysis of protein methylation (in particular arginine and lysine methylation) using LC tandem MS approaches presents a number of difficulties owing to the nature of the highly charged basic/hydrophilic peptides often generated from tryptic digests of methylated proteins. In this study, we have developed chromatographic separations at neutral $\mathrm{pH}$ using ammonium formate, which increases the retention and alters the selectivity of methylated peptides compared with traditional low $\mathrm{pH}$ reversed-phase separations. Therefore, such approaches allow the retention and separation of peptides that are not retained using conventional reversed-phase LC MS/MS analysis. We have also demonstrated, using a number of methylated proteins, including SFPQ, REF2-I, THOC4, MBP, Cren7, and Sul7D, that ETD offers significant advantages compared with CID for unambiguous assignment of protein methylation (arginine and lysine). The majority of methylated peptides identified had a predominant charge state +3 or greater under reversed-phase conditions. Therefore, as expected, ETD tandem MS generated increased backbone cleavage and improved tandem MS information compared with CID fragmentation of such peptides, enabling assignment of the site(s) of methylation in a wide range of peptides that was not possible from the
CID spectra alone. CID of arginine methylated peptides predominantly results in side-chain fragmentation, whereas CID of lysine methylated does not result in side-chain fragmentation and thus gives interpretable spectra. During ETD, side-chain fragmentation is largely avoided and, therefore, ETD is more beneficial for arginine methylated peptides compared to lysine methylated peptides.

Despite this, significant amounts of neutral losses for ETD of arginine methylated peptides were generated. This allowed for the sequence determination and the assignment of the type of methylation on the basis of the ETD spectra alone. The LC MS/MS approaches developed in this study offer significant advantages for future global studies that aim to further identify and characterize the emerging methylproteome.

\section{Acknowledgments}

The authors acknowledge support for this work by the Biotechnology and Biological Sciences Research Council UK [BB/ D011795/1] and the Engineering and Physical Sciences Research Council UK [EP/E036252/1]. A.P.L.S. is a RCUK Academic Fellow.

\section{Appendix A Supplementary Material}

Supplementary material associated with this article may be found in the online version at doi:10.1016/ j.jasms.2009.09.010.

\section{References}

1. Paik, W. K.; Paik, D. C.; Kim, S. Historical Review: The Field of Protein Methylation. Trends Biochem Sci. 2007, 32(3), 146-152.

2. Pahlich, S.; Zakaryan, R. P.; Gehring, H. Protein Arginine Methylation: Cellular Functions and Methods of Analysis. Biochim. Biophys. Acta 2006, 1764(12), 1890-1903.

3. Lake, A. N.; Bedford, M. T. Protein Methylation and DNA Repair. Mutat. Res. 2007, 618(1/2), 91-101.

4. Bedford, M. T.; Richard, S. Arginine Methylation an Emerging Regulator of Protein Function. Mol. Cells 2005, 18(3), 263-272.

5. Klose, R. J.; Yamane, K.; Bae, Y.; Zhang, D.; Erdjument-Bromage, H.; Tempst, P.; Wong, J.; Zhang, Y. The Transcriptional Repressor JHDM3A Demethylates Trimethyl Histone H3 Lysine 9 and Lysine 36. Nature 2006, 442(7100), 312-316. 
6. Cloos, P. A.; Christensen, J.; Agger, K.; Maiolica, A.; Rappsilber, J.; Antal, T.; Hansen, K. H.; Helin, K. The Putative Oncogene GASC1 Demethylates Tri- and Dimethylated Lysine 9 on Histone H3. Nature 2006, 442(7100), 307-311.

7. Chang, B.; Chen, Y.; Zhao, Y.; Bruick, R. K. JMJD6 is a Histone Arginine Demethylase. Science 2007, 318(5849), 444-447.

8. Cuthbert, G. L.; Daujat, S.; Snowden, A. W.; Erdjument-Bromage, H. Hagiwara, T.; Yamada, M.; Schneider, R.; Gregory, P. D.; Tempst, P.; Bannister, A. J.; Kouzarides, T. Histone Deimination Antagonizes Arginine Methylation. Cell 2004, 118(5), 545-553.

9. Wang, Y.; Wysocka, J.; Sayegh, J.; Lee, Y. H.; Perlin, J. R.; Leonelli, L.; Sonbuchner, L. S.; McDonald, C. H.; Cook, R. G.; Dou, Y.; Roeder, R. G. Clarke, S.; Stallcup, M. R.; Allis, C. D.; Coonrod, S. A. Human PAD4 Regulates Histone Arginine Methylation Levels via Demethylimination. Science 2004, 306(5694), 279-283.

10. Klose, R. J.; Zhang, Y. Regulation of Histone Methylation by Demethylimination and Demethylation. Nat. Rev. Mol. Cell Biol. 2007, 8(4), 307-318.

11. Smith, B. C.; Denu, J. M. Chemical Mechanisms of Histone Lysine and Arginine Modifications. Biochim. Biophys. Acta 2009, 1789(1), 45-57.

12. Guo, L.; Feng, Y.; Zhang, Z.; Yao, H.; Luo, Y.; Wang, J.; Huang, L. Biochemical and Structural Characterization of Cren7, a Novel Chromatin Protein Conserved Among Crenarchaea. Nucleic Acids Res. 2008, 36(4), 1129-1137.

13. Choli, T.; Henning, P.; Wittmann-Liebold, B.; Reinhardt, R. Isolation, Characterization, and Microsequence Analysis of a Small Basic Methylated DNA-Binding Protein from the Archaebacterium. Sulfolobus solfataricus. Biochim. Biophys. Acta 1988, 950(2), 193-203.

14. Cameron, D. M.; Gregory, S. T.; Thompson, J.; Suh, M. J.; Limbach, P. A.; Dahlberg, A. E. Thermus Thermophilus L11 Methyltransferase, PrmA, is Dispensable for Growth and Preferentially Modifies Free Ribosomal Protein L11 Prior to Ribosome Assembly. J. Bacteriol. 2004, 186(17), 5819-5825.

15. Boisvert, F. M.; Cote, J.; Boulanger, M. C.; Richard, S. A Proteomic Analysis of Arginine-Methylated Protein Complexes. Mol. Cell. Proteom. 2003, 2(12), 1319-1330.

16. Gehrig, P. M.; Hunziker, P. E.; Zahariev, S.; Pongor, S. Fragmentation Pathways of N(G)-Methylated and Unmodified Arginine Residues in Peptides Studied by ESI-MS/MS and MALDI-MS. J. Am. Soc. Mass Spectrom. 2004, 15(2), 142-149.

17. Rappsilber, J.; Friesen, W. J.; Paushkin, S.; Dreyfuss, G.; Mann, M. Detection of Arginine Dimethylated Peptides by Parallel Precursor Ion Scanning Mass Spectrometry in Positive Ion Mode. Anal. Chem. 2003, 75(13), 3107-3114.

18. Ong, S. E.; Mittler, G.; Mann, M. Identifying and Quantifying In Vivo Methylation Sites by Heavy Methyl SILAC. Nat. Methods 2004, 1(2), 119-126.

19. Brame, C. J.; Moran, M. F.; McBroom-Cerajewski, L. D. A Mass Spectrometry Based Method for Distinguishing Between Symmetrically and Asymmetrically Dimethylated Arginine Residues. Rapid Commun. Mass Spectrom. 2004, 18(8), 877-881.

20. Zhang, K.; Yau, P. M.; Chandrasekhar, B.; New, R.; Kondrat, R.; Imai, B. S.; Bradbury, M. E. Differentiation Between Peptides Containing Acetylated or Trimethylated Lysines by Mass Spectrometry: An Application for Determining Lysine 9 Acetylation and Methylation of Histone H3. Proteomics 2004, 4(1), 1-10.

21. Couttas, T. A.; Raftery, M. J.; Bernardini, G.; Wilkins, M. R. Immonium Ion Scanning for the Discovery of Post-Translational Modifications and Its Application to histones. J. Proteome Res. 2008, 7(7), 2632-2641.

22. Liu, Q.; Dreyfuss, G. In Vivo and In Vitro Arginine Methylation of RNA-Binding Proteins. Mol. Cell. Biol. 1995, 15(5), 2800-2808.
23. Wooderchak, W. L.; Zang, T.; Zhou, Z. S.; Acuna, M.; Tahara, S. M. Hevel, J. M. Substrate Profiling of PRMT1 Reveals Amino Acid Sequences that Extend Beyond the "RGG" Paradigm. Biochemistry 2008, $47(36), 9456-9466$.

24. Syka, J. E.; Coon, J. J.; Schroeder, M. J.; Shabanowitz, J.; Hunt, D. F. Peptide and Protein Sequence Analysis by Electron Transfer Dissociation Mass Spectrometry. Proc. Natl. Acad. Sci. U.S.A. 2004, 101(26), 9528-9533.

25. Mikesh, L. M.; Ueberheide, B.; Chi, A.; Coon, J. J.; Syka, J. E.; Shabanowitz, J.; Hunt, D. F. The Utility of ETD Mass Spectrometry in Proteomic Analysis. Biochim. Biophys. Acta 2006, 1764(12), 1811-1822.

26. Medzihradszky, K. F.; Zhang, X.; Chalkley, R. I.; Guan, S.; McFarland, M. A.; Chalmers, M. J.; Marshall, A. G.; Diaz, R. L.; Allis, C. D. Burlingame, A. L. Characterization of Tetrahymena Histone H2B Variants and Post-Translational Populations by Electron Capture Dissociation (ECD) Fourier Transform Ion Cyclotron Mass Spectrometry (FTICR MS). Mol. Cell. Proteom. 2004, 3(9), 872-886.

27. Taverna, S. D.; Ueberheide, B. M.; Liu, Y.; Tackett, A. J.; Diaz, R. L.; Shabanowitz, J.; Chait, B. T.; Hunt, D. F.; Allis, C. D. Long-Distance Combinatorial Linkage Between Methylation and Acetylation on Histone H3 N Termini. Proc. Natl. Acad. Sci. U.S.A. 2007, 104(7), 2086-2091.

28. Young, N. L.; Dimaggio, P. A.; Plazas-Mayorca, M. D.; Baliban, R. C. Floudas, C. A.; Garcia, B. A. High-Throughput Characterization of Combinatorial Histone Codes. Mol. Cell. Proteom. 2009, 8(10), 2266-2284.

29. Zhang, L.; Freitas, M. A. Comparison of Peptide Mass Mapping and Electron Capture Dissociation as Assays for Histone Post-Translationa Modifications. Int. J. Mass Spectrom. 2004, 234(1/3), 213-225.

30. Wang, H.; Straubinger, R. M.; Aletta, J. M.; Cao, J.; Duan, X.; Yu, H.; Qu, J. Accurate Localization and Relative Quantification of Arginine Methylation Using Nanoflow Liquid Chromatography Coupled to Electron Transfer Dissociation and Orbitrap Mass Spectrometry. J. Am. Soc. Mass Spectrom. 2009, 20(3), 507-519.

31. Vagin, V. V.; Wohlschlegel, J.; Qu, J.; Jonsson, Z.; Huang, X.; Chuma, S.; Girard, A.; Sachidanandam, R.; Hannon, G. J.; Aravin, A. A. Proteomic Analysis of Murine PIWI Proteins Reveals a Role for Arginine Methylation in Specifying Interaction with Tudor Family Members. Genes Dev. 2009, 23(15), 1749-1762.

32. Hanna, C.; Gjerde, D.; Nguyen, L.; Dickman, M.; Brown, P.; Hornby, D. Microscale Open-Tube Capillary Separations of Functional Proteins. Anal. Biochem. 2006, 350(1), 128-137.

33. Hautbergue, G. M.; Hung, M. L.; Golovanov, A. P.; Lian, L. Y.; Wilson, S. A. Mutually Exclusive Interactions Drive Handover of mRNA from Export Adaptors to TAP. Proc. Natl. Acad. Sci. U.S.A. 2008, 105(13), 5154-5159.

34. Tholey, A.; Toll, H.; Huber, C. G. Separation and Detection of Phosphorylated and Nonphosphorylated Peptides in Liquid ChromatographyMass Spectrometry Using Monolithic Columns and Acidic or Alkaline Mobile Phases. Anal. Chem. 2005, 77(14), 4618-4625.

35. Toll, H.; Oberacher, H.; Swart, R.; Huber, C. G. Separation, Detection, and Identification of Peptides by Ion-Pair Reversed-Phase High-Performance Liquid Chromatography-Electrospray Ionization Mass Spectrometry at High and Low pH. J. Chromatogr. A 2005, 1079, (1/2)274-286.

36. Panja, S.; Nielsen, S. B.; Hvelplund, P.; Turecek, F. Inverse Hydrogen Migration in Arginine-Containing Peptide Ions Upon Electron Transfer. J. Am. Soc. Mass Spectrom. 2008, 19(12), 1726-1742.

37. Xia, Y.; Han, H.; McLuckey, S. A. Activation of Intact Electron-Transfer Products of Polypeptides and Proteins in Cation Transmission Mode Ion/Ion Reactions. Anal. Chem. 2008, 80(4), 1111-1117.

38. Kim, S.; Lim, I. K.; Park, G. H.; Paik, W. K. Biological Methylation of Myelin Basic Protein: Enzymology and Biological Significance. Int. J. Biochem. Cell. Biol. 1997, 29(5), 743-751. 